\title{
Cross-Docking Operations for Supply Chain Logistics in JIT Production and Distribution Systems
}

\author{
Gyan Bahadur Thapa ${ }^{1}$, Tanka Nath Dhamala ${ }^{2}$, Shankar Raj Pant ${ }^{2}$ \\ ${ }^{1}$ Department of Mathematics, Pulchowk Campus, IOE, Tribhuvan University, Kathmandu, Nepal \\ ${ }^{2}$ Central Department of Mathematics, IOST, Tribhuvan University, Kathmandu, Nepal \\ Corresponding author: thapagbt@yahoo.com; thapaght@ioe.edu.np
}

\begin{abstract}
The multi-level production problem is one of the challenging research areas in supply chain management. We present brief literature review and mathematical models of multi-level just-in-time sequencing problem with a view of cross-docking approach for supply chain logistics. Describing cross-docking operations, we propose a mathematical model for the cross-docking supply chain logistics problem to minimize the operation time as truck sequencing problem. We establish a proposition as the synthesis of the production and logistics.
\end{abstract}

Key Words: Just-in-time, supply chain, logistics, cross-dock, operation time.

\section{Introduction}

Just-in-time (JIT) production system is a management philosophy that uses a set of integrated activities to achieve manufacturing flexibility with minimum shortages and inventories, which was first practiced and perfected by Toyota motor company in Japan around 1970s. Moreover, $J I T$ is a comprehensive philosophy that advocates for planned elimination of waste, simplification of operations, continuous improvement of productivity, customer satisfaction, standardized work and employee involvement among many other things. The Toyota production system (TPS) is well-presented by Toyota production house in Fig. 1.1. One of the manufacturing problems that are often associated with JIT practices is sequencing mixed models on assembly lines, which is widely used in manufacturing industries to meet diversified demand of consumers without the need for large product inventories. Sequencing products to be assembled at the mixed model assembly line is recognized as an important work for improving its performance. The production and distribution processes are the counterparts of each other. Balanced distribution system helps to reduce inventories.

A recent trend in the domain of production management is the integration of overall production processes within and among the companies. A synchronized view of these processes includes all traditional areas of supplier-buyer relationships coping with the production of goods and their distribution as well, in particular production and logistics. Traditional manufacturing operations produce goods in advance of demand to have products in place when demand occurs. Parts, components, materials, subassemblies, and finished products are made in their work centers and pushed down to the next operation in the manufacturing sequence. This type of flow control is 
referred as a push system. In contrast, JIT manufacturing operations produce goods only when they are needed, where they are needed and how much they are needed.



If any product is not demanded by a customer, then it is not produced. This type of flow control is referred as a pull system. Thus, JIT production system (JITPS) is a pull production system (Fig. 1.2) where products are assembled just before they are sold; subassemblies are made just before the products are assembled and the components are fabricated just before the subassemblies are made.

Fig. 1.1 Toyota Production House



Fig. 1.2 Pull Production System

Moreover, JITPS is a management philosophy of continuous improvement including three sequential components: people involvement $\rightarrow$ total quality control $\rightarrow$ JIT flow; jointly called productivity triad [18]. Sequenced delivery of the materials and products throughout overall supply chain of manufacturing companies is the ultimate realization of JIT principles- zero inventories, zero defects, zero waste. Some benefits of JITPS are reduced inventory, improved quality, shorter lead times, lower production costs, increased productivity, increased machine utilization, increased workforce flexibility and greater output volume flexibility. The two types of 
$J I T$ sequencing problems are studied in the literature: single-level [7] and multi-level $[9,16]$. The single-level problem is to minimize the variations in the product rates at which different products are produced on the production line, called product rate variation $(P R V)$ problem. The multi-level problem is to minimize the variations in demand rates for outputs of supplying processes, called output rate variation $(O R V)$ problem.

Supply chain logistics is the task of integrating organizational units along a supply chain and coordinating materials, information and financial flows in order to fulfill final customer demands with the aim of improving competitiveness of the manufacturing company as a whole. This is best visualized by the house of supply chain management and logistics in [19]. To realize the best quality production and timely distribution for the customer in a rapidly changing technical environment, it is essential to create a cross-docking environment throughout the whole supply chain system that is capable to address the diversified demands. Cross-docking is the movement of products directly from receiving dock to shipping dock with minimum dwell time in between. By arranging for immediate cross-docking of incoming products, retailers are able to reduce to a minimum in-transit time for their incoming products. Moreover, cross-docking is a relatively new logistics technique used in the retail and trucking industries with operations seeking to move materials from inbound locations to outbound locations as quickly as possible. However, crossdocking operations require good information systems and close synchronization of all inbound and outbound shipments. The mutual coordination among independent firms (viz., raw-material suppliers, manufacturers, distributors and retailers) is the crux to attain the flexibility required to enable them in the progressive improvement of logistics processes in response of rapidly changing market conditions.

Rest of the paper is planned as follows: Section 2 gives brief literature surveys of JITPS as ORV $P R V$ problems and of cross-docking operations in the supply chain logistics. Section 3 formulates the $O R V$ problem. Sections 4 and 5 provide the model description and model formulation of cross-docking supply chain logistics problem. Finally, section 6 concludes the paper.

\section{Literature Reviews of JITPS and Cross-Docking Operations}

The single-level JIT sequencing problem aims to minimize the maximum deviation and the total deviation between the actual and ideal productions. The $P R V$ problem is wisely studied in $[7,8]$ providing a polynomial time solution in terms of assignment formulation. A recent survey of $P R V$ problem with the approach of discrete apportionment can be found in $[18,20]$. Another review on sequencing approaches for mixed-model JITPS can be found in [4]. An efficient frontier is established for sum deviation JIT sequencing problem via apportionment in [3]. The single-level problem is extended into the multi-level $[12,16]$, which deals with several levels such as raw materials $\rightarrow$ components $\rightarrow$ subassembly $\rightarrow$ final product $\rightarrow$ distribution centers $\rightarrow$ retailers $\rightarrow$ customers [19]. Most discrete manufacturing systems are multi-level in nature, characterized by the condition where several parts are used to produce a particular part at a higher level, terminating at the last level yielding the final product with direct consumer utility. The sequence of products on the final assembly line impacts greatly on inventory levels of parts used directly for assembly and other parts in the system. Recently, the problem of determining an appropriate product sequence has been attracting a lot of attention. The $O R V$ problem is proved $N P$-hard, even in special cases $[7,9]$. However, the dynamic programming solution is proposed in $[9,13]$. The $O R V$ problem with pegging assumption is effectively solved in [16], which reduced the problem into the weighted $P R V$ problem. Modifying the solution techniques used for 
the unweighted single-level problem, the pegged multi-level problem may be solved to optimum in time which is polynomial in the total product demand and the weighted factors.

The JIT production logistics forms a specific part of the supply chain, which deals with the planning and control of materials and information flows throughout the production and distribution supply chains of manufacturing companies with the mission to get the right materials to the right place at the right time in perfect quality at the lowest possible costs $[5,18]$. The JIT logistics is performed to optimize some sort of given performance measures, such as to minimize total operating costs, transportation costs, operating time and to satisfy a given set of constraints. Moreover, it is a mobility concept relating with tangible as well as intangible assets. Thus, it is the true realization of JIT production and delivery systems: a management philosophy which uses a set of integrated activities to achieve manufacturing flexibility with minimum shortages and inventories. As its pull nature, the JITPS starts a supplying process only when a consuming process demands the supplying process. The role of information logistics in supply chain production process is briefly studied in [17]. The extended scenario of the supply chain network in multi-level production system is shown in Fig. 2.1 in terms of inbound logistics and outbound logistics.

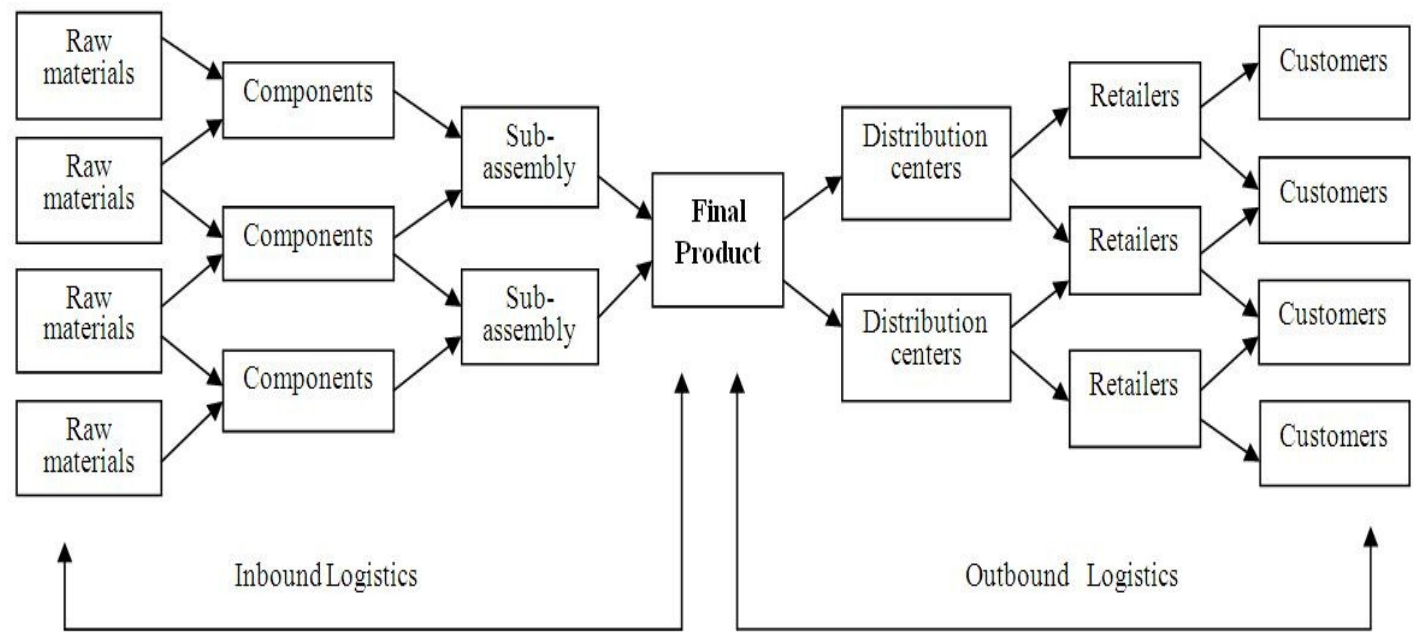

Extended supply chain network

Fig. 2.1 Supply Chain Synchronization in Production and Logistics

As a dynamic JIT distribution centre, cross-docking has been widely applied in both manufacturing systems and logistics, since cross-docking operation (CDO) favors the timely distribution of freight and a better synchronization with the demand to provide improved customer service. Perishable products reach the marketplace faster, preserving quality and freshness. $C D O$ is considered as the best method to reduce inventory and to improve responsiveness of various customer demands. In addition of previous studies dealt mostly with the conceptual advantages of $C D O$ or actual issues from the strategic viewpoint, it is also necessary to consider cross-docking from an operational viewpoint in order to find the optimal vehicle routing schedule. $C D O$ is a process where products are received in a distribution center occasionally merged with other products going to the same destination, then shipped at the earliest opportunity. Cross-docking supply chain logistics (CDSCL) system is a material handling 
and distribution concept in which the products move directly from receiving dock to shipping dock, without being stored in a warehouse or distribution center [19]. Buffa [2] showed that logistics cost could be reduced by integrating the inbound and outbound vehicles in the distribution system. Uday and Viswanathan [22] provided a framework for understanding and designing cross-docking systems and discussed techniques that can improve the overall efficiency of the logistics and distribution operations. The advantages of $C D O$ include minimal inventory, low handling costs, low space requirement, centralized processing and low transportation costs. The functional activities at cross-docking centers $(C D C)$ are presented in Fig. 2.2 and Fig. 2.3. Only few research papers deal with the short-term scheduling problems arising from the daily operations of cross-docking terminals. Material handling inside the crossdock terminal for a given truck schedule is considered in [11].

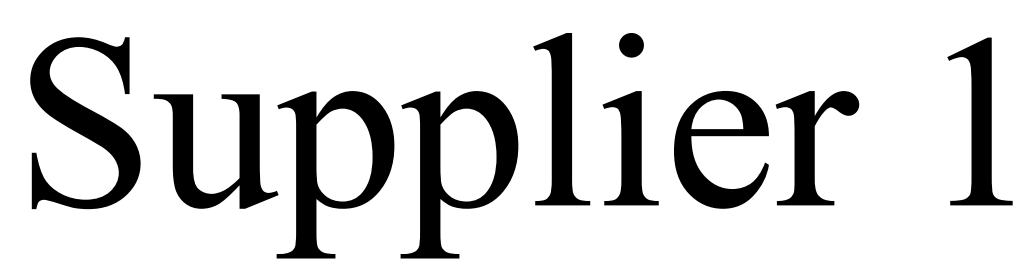

Fig. 2.2 Freight Consolidation at the Transfer Terminal

Once a set of inbound and outbound trucks is docked, jobs consisting of products to be handled have to be assigned to resources, i.e., workers and means of conveyance like fork-lifts, in such a way that efficient unloading, sorting and loading operations render possible. It is modeled as a machine scheduling problem and proposed a meta-heuristic suited for its solution [10]. A specific truck scheduling problem is covered at a parcel hub and solved by a simulation-based optimization approach [11]. A special kind of cross-dock terminal with a conveyor belt system is treated in [23] where the transportation of goods within the dock is modeled as a detailed scheduling problem providing a priority rule based start heuristic. Boysen et al. [1] treated a stylized one inbound door serves one outbound door setting in order to generate fundamental insights to the underlying real-world problem structure.

Exact handling times for inbound trailers depend on the exact packing of goods and the sequence in which they can be obtained, whereas those for outbound trailers have to account for load stability and the sequence in which customers are served. Furthermore, the determination of transportation times between doors results to a complex optimization problem in itself. Thus, handling times used in a detailed truck scheduling model are merely estimated average times and often bound to heavy inaccuracies. Under such prerequisites, detailed models may lead to more misleading or even infeasible plans when compared to aggregate models. So they merged individual handling times for products to service slots to which inbound and outbound trucks are assigned. A slot comprises the time required for completely unloading an inbound truck and completely loading an outbound truck respectively. Handling times in between dock doors are 
considered by a delay which covers the time span until incoming products are available at an outbound door. By a simultaneous scheduling of inbound and outbound trucks, incoming flows of products are harmonized with outbound flows, so that a JIT supply of products, and thus, a reduced turnover time is enabled.

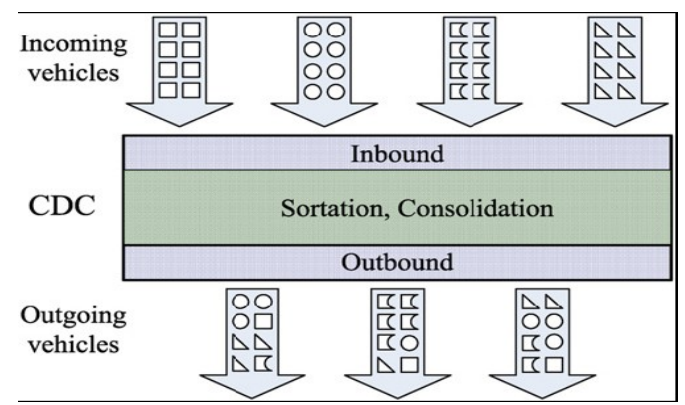

Fig. 2.3 Cross-Docking Operational Scenario

\section{ORV Problem Formulation}

Assume that the mixed-model multi-level JITPS consist of $L$ levels of manufacturing operations, indexed by $l=1,2, \ldots, \quad L$ with the first product level 1 . The number of different part types and the demand of item $i$ in level $l$ are denoted by $n_{l}$ and $d_{i l}$ respectively, where $i=1,2, \ldots, n_{l}$. The number of total units of item $i$ at level $l$ required to produce one unit of product $p$ is denoted by $t_{i l p}$ such that $d_{i l}=\sum_{p=1}^{n_{l}} t_{i l p} d_{p 1}$ is the dependent demand for item $i$ at level $l$ determined by the final product demands $d_{p 1}, p=1,2, \ldots, n_{l}$ and $l=1,2, \ldots, \mathrm{L}$. Note that $t_{i p}=1$ if $\mathrm{i}=p$ and 0 if otherwise. Finally, $D_{l}=\sum_{i=1}^{n_{1}} d_{i l}$ denotes the total demand at level $l$, and the ratio $r_{i l}=$ gives the demand rate for item $i$ of level $l$ such that $\sum_{i=1}^{n_{l}} r_{i l}=1$ at each level.

It is noteworthy that the model of $O R V$ problem is assumed to be non-preemptive- once commenced production of a product at level 1 must be completed prior to switch into another. This creates the concept of various stages or cycles in the production system. The production schedule at level 1 consists of $D_{1}$ stages in total and at each stage a single unit of an end-product can be processed. An item is said to be in stage $k,\left(k=1,2, \ldots, D_{1}\right)$, if $k$ units of product have been produced at level 1 and there will be $k$ complete units of various products $p$ at level 1 during the first $k$ time units. 
Let $x_{i l k}$ be the necessary quantity of item $i$ produced at level $l$ during the time units 1 through $k$ and $y_{l k}=\sum_{i=1}^{n_{l}} x_{i l k}$ be the cumulative quantity of item $i$ produced at level $l$ during the same time units such that $y_{1 k}=\sum_{i=1}^{n_{l}} x_{i 1 k}=k$. Due to the pull nature of the JITPS, the particular combination of the highest level products produced during the $k$ time units (the $x_{p 1 k}$ values) determines the necessary cumulative production at every other level. Thus, the required cumulative production for item $i$ at level $l$ with $l \geq 2$ through $k$ time units is given by $x_{i l k}=\sum_{p=1}^{n_{l}} t_{i l p} x_{p 1 k}$. For a unimodal convex penalty function $F_{i}, i=1,2, \ldots, n_{l}$ with minimum 0 at 0 , the maximum and sum deviation multi-level JIT sequencing (i.e.,ORV) problems in mixed-model systems are formulated to minimize the objectives $Z_{\max }$ and $Z_{\text {sum }}$ as the followings [6, 12]:

$$
\begin{array}{r}
Z_{\max }=\min _{i, l, k} \max F_{i}\left(x_{i l k}-y_{l k} r_{i l}\right) \\
Z_{\text {sum }}=\min \sum_{k=1}^{D_{1}} \sum_{l=1}^{L} \sum_{i=1}^{n_{l}} F_{i}\left(x_{i l k}-y_{l k} r_{i l}\right)
\end{array}
$$

subject to

$$
\begin{gathered}
x_{i l k}=\sum_{p=1}^{n_{l}} t_{i l p} x_{p 1 k}, \quad i=1,2, \ldots, n_{l} ; l=1,2, \ldots, L ; k=1,2, \ldots D_{1} \\
y_{l k}=\sum_{i=1}^{n_{l}} x_{i l k}, \quad l=2,3, \ldots, L ; k=1,2, \ldots, D_{1} \\
y_{1 k}=\sum_{p=1}^{n_{1}} x_{p 1 k}=k, k=1,2, \ldots, D_{1} \\
x_{p 1 k} \geq x_{p 1, k-1}, p=1,2, \ldots, n_{1} ; k=1,2, \ldots, D_{1} \\
x_{p 1 D_{1}}=d_{P 1} ; x_{p 10}=0, p=1,2, \ldots, n_{1} \\
x_{i l k} \geq 0 \in Z^{+}, i=1,2, \ldots, n_{1} ; l=1,2, \ldots L ; k=1,2, \ldots, D_{1}
\end{gathered}
$$

The objective functions (1) and (2) minimize the maximum and sum deviation measures respectively. The constraint (3) ensures that the necessary cumulative production of part $i$ at level $l$ by the end of time unit $k$ is determined explicitly by the quantity of products produced at level 1. Constraints (4) and (5) show the total cumulative production of level $l$ and level 1 respectively during the time slots 1 through $\mathrm{k}$. Constraint (6) ensures that the total production of every product over $k$ time units is a non-decreasing function of $k$. Constraint (7) guarantees that the demands for 
each product are met exactly. Constraints (5), (6), (8) jointly ensure that exactly one unit of a product is scheduled during one time unit in the product level. The particular cases of the ORV objectives (1) and (2) are studied in the literature as the absolute and the squared deviation objectives in both maximum and sum deviation cases $[4,21]$.

Being $N P$-hard in nature, there is no efficient algorithm for the above general $O R V$ objectives with the assigned constraints therewith. The only published algorithm for scheduling mixedmodel multi-level JIT production systems is the goal-chasing method (GCM), developed and used by Toyota to schedule automobile final assembly lines [15]. This algorithm considers only two levels- final assembly and sub-assemblies. We refer [12, 15], for the extensive study of $G C M$ and extended GCM. We consider the cross-docks in between each level so that the operation time is minimized and inventory level is reduced.

\section{CDSCL Model Description}

The production logistics problem is considered as truck sequencing problem over here. The notational convention is described as follows: Let $I$ and $\mathrm{O}$ be the sets of inbound and outbound trucks at the single receiving door and the single shipping door respectively of the cross-docking terminal. Each inbound truck is loaded with units of different products $p \in P$. Suppose $\alpha_{\text {ap }}$ be the number of units of product type $p$ arriving in an inbound truck $\alpha$ and $b_{\beta p}$ be the number of product type $p$ to be loaded onto outbound truck $\beta$. All product units are completely unloaded within a service slot $t$ to which the respective inbound truck is assigned, so that all handling operations (e.g. docking, unloading and undocking) required to process the truck are executed within this time span, e.g., an hour or two. Moreover, all inbound trucks are assumed to be available for processing at the beginning of the planning horizon, so that a static problem with identical arrival dates of inbound trucks is considered. The assumption of equally long service times can be seen as a reasonable approximation of reality, whenever vehicle capacities and the number of products per vehicle do not strongly differ. As trailers are typically of a standardized size and cross-docking aims at moving only full truck loads, this premise is fulfilled whenever all processed products are of comparable size (e.g. mail distribution centers) or all truck loads resemble a representative average truck load (e.g., rotational deliveries of special promotional offers to all stores of a retail chain).

Once unloaded, the delivered products have to undergo several subsequent operations before they are available for being loaded onto the outbound trucks at the shipping door. These operations include recording of any product unit in the information system, examining the product correctness and quality, collecting, sorting, rearranging and packing to recombine products from different inbound trucks to form the load of a certain outbound truck. Finally, the products have to be transported to the shipping door, where they wait in an intermediate buffer of sufficient size until they are needed. This variety of tasks from recording to transporting is assumed to last a fixed movement time $m$ irrespective of the truck load actually processed. Then, all products arriving in a slot $t$ are available for loading at the shipping dock not before slot $t+m$ if the movement process can be started for any unloaded unit immediately, e.g. when applying a conveyor belt system. If the movement starts not before the complete inbound truck has been unloaded completely (e.g., a worker stacks all units behind the receiving door before moving them), the units are first available at slot $t+m+1$. However, the displacement $m$ or $m+1$, respectively, can be ignored (set to zero) when modeling and solving the problem, because after 
having determined a solution, an appropriate re-indexing of slots outbound trucks are assigned to allow the exact determination of the outbound schedule. Similarly to constant unloading times, it is assumed that the movement time $m$ is independent of the inbound truck and the loaded products, because handling full truck loads, which may always consist of almost the same number of product units, should take very similar times.

The set $O$ of outbound trucks is to be loaded at the shipping door for each $\beta \in O$ with a

predetermined number of units $\mathrm{b}_{\beta \mathrm{p}}$ of the different products $p \in P$. Also, it is assumed that all

handling operations per truck are completed within a single slot. An outbound truck can be assigned to a slot $t$ not before enough stock has accumulated in the intermediate buffer to serve all demanded product units of the truck. As only temporary stock is allowed (or desired) within a cross dock, it is assumed that temporary stock is empty before the first inbound truck arrives and is emptied out again after the last outbound truck was served. Thus, within our model the following equality holds: $\Sigma_{a \in I} a_{a p}=\sum_{\beta \in 0} b_{\beta p}, \forall p \in P$. The simplifying assumptions applied to our base model are described in [1].

\section{CDSCL Model Formulation}

We set the following notations to formulate the $C D S C L$ problem:

$I=$ Set of inbound trucks (indexed by $\alpha$ );

$O=$ Set of outbound trucks (indexed by $\beta$ )

$P=$ set of products (indexed by $p$ );

$T=$ total number of time slots (indexed by $t$ )

$a_{\alpha p}=$ quantity of $p$ arriving in truck $\alpha$;

$b_{\beta p}=$ quantity of $p$ to be loaded in truck $\beta$

$x_{\alpha t}=1$, if inbound truck $\alpha$ is assigned to slot $t$

$y_{\beta t}=1$, if outbound truck $\beta$ is assigned to slot $\mathrm{t}$

0 , if otherwise

0 , if otherwise

We define operation time of $\alpha$ trucks by $t x_{\alpha t}$ and operation time of $\beta$ trucks by $t y_{\beta t}$ in the same time slot $t$. As a direct result of the simplifying assumptions in [1], the inbound and outbound schedule can be readily derived by the sequence of inbound and outbound trucks, so that the problem reduces to a truck sequencing problem (TRSP). The objective is to sequence the trucks in such a way that the operation time is minimized which comprises the time span starting from the first slot to which an inbound truck is assigned and lasts until the final slot in which an outbound truck is processed. With the above notations, we formulate the TRSP problem in multilevel to minimize the operation time as follows:

$$
\begin{array}{ll}
\text { Minimize } & \mathrm{M}=\operatorname{Max}\left|t \mathrm{x}_{\alpha t}-t y_{\beta t}\right| \forall \alpha \in \mathrm{I} \text { and } \beta \in 0 \\
\text { subject to } & \sum_{t=1}^{T} x_{\alpha t}=1, \quad \forall \alpha \in I \\
& \sum_{\alpha \in I} x_{\alpha t} \leq 1, \quad \forall t=1,2, \ldots, T
\end{array}
$$




$$
\begin{aligned}
& \sum_{t=1}^{T} y_{\beta t}=1, \quad \forall \beta \in O \\
& \sum_{\beta \in O} y_{\beta t} \leq 1, \quad \forall t=1,2, \ldots, T \\
& \sum_{\tau=1}^{t} \sum_{\alpha \in I} x_{\alpha \tau} . a_{\alpha p} \geq \sum_{\tau=1}^{t} \sum_{\beta \in O} y_{\beta \tau} . b_{\beta p}, \forall t=1, \ldots, T ; p \in P \\
& x_{\alpha t} \in\{0,1\} \quad \forall \alpha \in I ; t=1, \ldots, T \\
& y_{\beta t} \in\{0,1\}, \quad \beta \in O ; t=1, \ldots, T
\end{aligned}
$$

The objective (9) minimizes the sum of the absolute difference of operation times of outbound trucks $\beta$ and inbound trucks $\alpha$. The constraint (10) ensures that each inbound truck is processed in exactly one slot, whereas (11) enforces that in each slot at most one inbound truck can be assigned. In analogy, these two conditions hold true for outbound trucks by constraints (12) and (13). Constraints (14) ensure that an outbound truck can only be assigned to a slot $t$ whenever all required products are available (delivered by preceding inbound trucks yet not consumed by preceding outbound trucks) to satisfy the demand for product units of each type $p$. So, the available stock accumulated by all inbound trucks assigned to slots $\tau=1,2, \ldots, t$ has to exceed the total demand for product units of outbound trucks scheduled up to the actual slot $t$ (recall that it will actually be slot $t+m$ or even $t+m+1$ when realizing the schedule). The constraints (15) and (16) represent the binary variables for inbound and outbound trucks respectively.

On providing the left shifting property, Boysen et al. [1] proved that the TRSP is NP-hard in the strong sense. The overall TRSP problem is decomposed into two sub-problems, namely inbound and outbound TRSPs, written as IBD-TRSP and $O B D-T R S P$ respectively. It is divided into subproblems by fixing a particular inbound (outbound) sequence and then finding the optimal outbound (inbound) sequence respectively. A comparison of IBD-TRSP and OBD-TRSP reveals that their mathematical structures are identical. As a consequence, any algorithm for $O B D-T R S P$ can be used to solve IBD-TRSP and vice versa. In fact, IBD-TRSP can be seen as a reverted $O B D-T R S P$, in the sense that the solution of an instance of IBD-TRSP with an algorithm designed for $O B D-T R S P$ requires the following steps: (a) Revert the given outbound sequence and set it as the modified inbound sequence. Consider the set of inbound trucks $I$ to be scheduled as the modified set of outbound trucks $O$, (b) Solve $O B D$-TRSP with the modified input data, and (c) The reverted optimal outbound sequence constitutes the optimal inbound sequence for the original IBD-TRSP instance.

An exact dynamic programming approach is introduced and a heuristic starting procedure is proposed to solve the identified sub-problems [1]. The algorithmic descriptions are limited to $O B D-T R S P$, as they are directly transferable to IBD-TRSP. We conjecture that the multi-level JIT production problem and the cross-docking supply chain logistics problem are counterparts of 
each others. In this regard, we have proposed the following proposition to integrate $O R V$ and CDSCL problems:

Proposition 1: The solution of $O R V$ problem is balanced iff the solution of $C D S C L$ problem is balanced.

\section{Conclusion}

We have given a mathematical model for minimization of operation time in terms of absolute difference of operation times of inbound and outbound trucks. Our model is slightly different comparing with the model of Boysen et al. [1] that considers the operation time of outbound trucks only. We have considered only the $O R V$ model and the logistics model in this paper. However, the proper coordination between multi-level production lines and distribution lines plays an important role to balance the overall supply chain management of the manufacturing companies. The simultaneous study of the multi-level JIT production problem and the CDSCL problem is our due course.

\section{REFERENCES}

[1] Boysen, N., Fliedner, M. and Scholl, A. (2010), Scheduling inbound and outbound trucks at cross docking terminals. Operations Research Spectrum, 32, 135-161

[2] Buffa, F.P. (1986), Inbound logistics: Analysing inbound consolidation opportunities. International Journal of Physical Distribution and Materials Management, 16, 3-32

[3] Dhamala, T. N., Thapa, G. B. and Yu, H. (2010), An efficient frontier for sum deviation just-in-time sequencing problem in mixed-model systems via apportionment. Accepted for publication in International Journal of Automation and Computing

[4] Dhamala, T. N. and Khadka, S. R. (2009), A review on sequencing approaches for mixed-model just-in-time production system. Iranian Journal of Optimization, 1, 266-290

[5] Ghiani, G., Laporte, G. and Musmanno, R. (2004), Introduction to logistics systems planning and control. John Wiley and Sons Ltd

[6] Kubiak W. and Sethi S. P. (1991), A note on level schedules for mixed-model assembly lines in just-in-time production systems. Management Science, 37(1), 121-122

[7] Kubiak, W. (1993), Minimizing variation of production rates in just-in-time systems: A survey. European Journal of Operational Research, 66, 259-271

[8] Kubiak, W. and Sethi, S. P. (1994), Optimal just-in-time schedules for flexible transfer lines. The International Journal of Flexible Manufacturing Systems, 6, 137-154

[9] Kubiak, W., Steiner, G. and Yeomans, J. S. (1997), Optimal level schedules for mixed-model multilevel just-in-time assembly systems. Annals of Operation Research, 69, 241-259

[10] Li, Y., Lim, A. and Rodrigues, B. (2004), Cross-docking - JIT scheduling with time windows. Journal of the Operations Research Society, 55, 1342-1351

[11] McWilliams, D. L., Stanfield, P. M. and Geiger, C. D. (2005), The parcel hub scheduling problem: A simulation-based solution approach. Computer Industrial Engineering, 49, 393-412

[12] Miltenburg, J. and Sinnamon, G. (1989), Scheduling mixed-model multi-level just-in-time production systems. International Journal of Production Research, 27, 1487-1509

[13] Miltenburg, J., Steiner, G and Yeomans, S. (1990), A dynamic programming algorithm for scheduling mixed-model JIT production systems. Mathematical and Computer Modeling, 13(3), 5766 
[14] Miltenburg, J. and Goldstein, T. (1991), Developing production schedules which balance part usage and smooth production loads for JIT production systems. Naval Research Logistics, 38, 893-910

[15] Monden, Y. (1983), Toyota Production Systems: Practical Approach to Production Management. Industrial Engineering and Management Press, Norcross, GA

[16] Steiner, G. and Yeomans, J. S. (1996), Optimal level schedules in mixed-model multi-level just-intime assembly systems with pegging. European Journal of Operational Research, 95, 38-52

[17] Thapa, G. B., Dhamala, T. N. and Yu, H. (2010), Role of informed logistics in supply chain production process. To appear in Springer book proceedings of HAM Conference, (May 13-14, 2010) UK

[18] Thapa, G. B. and Dhamala, T. N. (2009), JIT sequencing in mixed-model production systems relating with fair representation in apportionment theory. The Nepali Mathematical Sciences Report, 29 (1 \& 2), 29-68

[19] Thapa, G. B. and Dhamala, T. N. (2010), Cross-docking framework of supply chain logistics in justin- time production scheduling. Submitted to Journal of Institute of Science and Technology, TU, Nepal

[20] Thapa, G. B. and Dhamala, T. N. (2009), A synthetic study to minimize the inequality measures in just-in-time sequencing problem via optimization methods. Proceedings of the $5^{\text {th }}$ Asian Mathematical Conference (June, 2009), Kuala Lumpur, Malaysia

[21] Thapa, G. B., Dhamala, T. N. and Yu, H. (2010), Mathematical model of cross-docking operation in supply chain logistics under multi-level just-in-time production environment. The Nepali Mathematical Sciences Report, 30 (1\&2), 24-35

[22] Uday, M. A. and Viswanathan, S. (2000), Effective cross-docking for improving distribution efficiencies. International Journal of Logistics: Research and Applications, 33, 291-302

[23] Yu, W. and Egbelu, P. J. (2008), Scheduling of inbound and outbound trucks in cross docking systems with temporary storage. European Journal Operations Research, 184, 377-396 